\title{
Nephrotic syndrome associated with metastatic melanoma: case report
}

\author{
Fabiana Bienes ${ }^{1}$, Germana Brito ${ }^{2}$, Joubert Alves ${ }^{2}$, Aline Baptista ${ }^{2}$, Luis Andrade ${ }^{2}$, Marina \\ Imanishe $^{2}$, and BENEDITO PEREIRA ${ }^{2}$ \\ ${ }^{1}$ UNINOVE Curso de Medicina \\ ${ }^{2}$ ACCamargo Cancer Center International Research Center
}

July 29, 2021

\begin{abstract}
Nephrotic syndrome may be the first clinical manifestation of neoplasms, such as paraneoplastic syndrome. Several studies have been identified the association of cancer with nephrotic syndrome, although laboratory and histological markers that correlate the presence of NS and melanoma, are still needed.
\end{abstract}

\section{Introduction}

The expression is paraneoplastic syndrome defined by clinical manifestations that are not directly related to tumor burden, tumor metastasis or invasion, but as a result of the tumor cells secretions produced, such as cytokines, tumor antigens, hormones and growth factors. $\left(\begin{array}{l}1 \\ \mathbf{~ - 2}\end{array}\right)$ The tumor producing these substances can damage the kidney, generating paraneoplastic glomerulopathies. The prevalence of cancer in patients with nephrotic syndrome is $11-13 \%$, but this percentage varies according to the patient's age, especially those with more than 50 years, the sex male, which is more prevalent, the type of glomerulonephritis (GN), whose highest incidence occurs in membranous nephropathy, with the type of malignancy and the use of alkylating agents to treat glomerular disease, which may subsequently influence the development of malignancies. (3)

The tumors that are most associated with this type of paraneoplastic syndrome are carcinomas, mainly pulmonary and gastrointestinal tract, and those of hematopoietic origin, such as Hodgkin's lymphoma. Among the most common paraneoplastic glomerulopathy found: GN membranous, GN minimal injuries, focal and segmental glomerulosclerosis (FSGS), GN membranopoliferative, IgA nephropathy, and GN rapidly progressive, with the first four are manifested clinically as nephrotic syndrome. The NS is characterized by presenting clinical -laboratorial of: proteinuria greater than or equal to $3,5 \mathrm{~g} / 24 \mathrm{~h}$, hypoalbuminemia, hyperlipidemia and edema, with patients with this disease have a tendency to develop combination of hypertension and microscopic hematuria. $(1,4)$

The diagnosis of paraneoplastic glomerulopathy is based on the following criteria: lack of an alternative etiology associated with the syndrome, remission of clinical and histological manifestations after complete treatment of the neoplasia, whether performed by surgical removal of the tumor or chemotherapy, recurrence of neoplasia is accompanied by recurrence of glomerulopathy and there must be an association of pathophysiology between the two diseases. These criteria suggest the occurrence of paraneoplastic glomerulopathy, but confirmation of the diagnosis is acquired by renal biopsy, which also helps determine the patient's prognosis and treatment. $(1,2,3,5)$

The occurrence of glomerulonephritis may occur before, concurrent or after the diagnosis of cancer and a temporal relationship is suspected when proteinuria occurs six months before or after the diagnosis of 
malignancy, but emergence of the risk the cancer persists for more than 10 years after Diagnostic confirmation of glomerulopathy. $(\mathbf{1}, \mathbf{2 , 5 , 6 )}$ Therefore, it is extremely important to research neoplasms in patients with idiopathic nephrotic syndrome, especially in those over 50 years of age. And assessment should be directed according to history, physical examination, personal and family history of cancer. We also consider neoplasms related frequently nephrotic syndrome and invested initial should be carried out screening of radiography or computed tomography, colonoscopy, prostate specific antigen, mammography, among others. Even if these tests are unchanged, the patient should often be followed for a long time, due to the long period of risk of cancer. $(1,7)$

Paraneoplastic membranous nephropathy (MN) is the most common form of nephrotic syndrome in adults, with males accounting for $70 \%$ of cases. Its prevalence in malignancies is from 2 to $11 \%$ and in patients over 60 years and this rate can reach $22 \%$, in smokers (20 packs / year) this prevalence is also increased. (1, 2, $\mathbf{8}$, 9)Studies have shown evidence showing differences in the pathophysiology of primary paraneoplastic membranous nephropathy. The phospholipase A2 receptor (PLA2R) was identified as the primary target antigens involved in most primary MN adult, producing self - antibodies anti-PLA2R and immune deposits glomerular mainly consist of IgG4 subclass, the MN paraneoplastic these Anti-PLA2R autoantibodies are rarely observed, immune deposits in the glomerulus are particularly characterized by IgG1 and IgG2 subclasses, and the presence of more than eight inflammatory cells infiltrating the glomerulus increases the likelihood of being a paraneoplastic MN with $92 \%$ sensitivity and $75 \%$ specificity. Complete tumor resolution through surgery, chemotherapy and/or radiotherapy promotes NM remission with proteinuria remission. $(\mathbf{7}, \mathbf{1 0})$

There are other less frequent associations of para-neoplastic syndrome with glomerular changes. Among these is the minimal change disease, the membranopoliferative glomerulonephritis, rapidly progressive glomerulonephritis paraneoplastic, Iga nephropathy and focal and segmental glomerulosclerosis (FSGS).

Paraneoplastic minimal lesion disease is more associated with Hodking's lymphoma, but there are also reported cases of leukemia and carcinomas (lung, kidney, gastrointestinal tract, ovarian, mesothelioma), in which in most cases it is diagnosed concomitantly with the diagnosis of malignancy. This disease is histologically defined by normal light microscopy, negative immunofluorescence and electron microscopy, which diffuses the foot processes diffusely. $(4,11)$ Its pathophysiology can be explained by an intense inflammatory reaction in response to the tumor, evolving to formation of granulomas with production of cytokines and growth factors (mainly VEGF - vascular endothelial growth factor) which may promote increased permeability of the glomerular basement membrane, allowing the passage of proteins. In addition to treating malignancy, the use of corticosteroids is very effective in resolving this type of glomerulopathy. $(\mathbf{1 0 , 1 2 )}$

The membranoproliferative GN paraneoplastic occurs primarily in lymphoproliferative malignancies but can also occur in carcinomas such as smalls cells. It is histologically characterized by mesangial proliferation, thickening of the capillary wall due to enlargement of the subendothelial mesangium and cellular enlargement consisting of mesangial cells and infiltrating leukocytes. (3) There are three types of histological classification, types I and III derive from changes in the immune complex, and type II derive from modifications in the complement pathways. The pathogenesis of this disease is not well understood, several studies have shown glomerular deposits containing specific tumor antigens and their antibodies and cryoglobulins appear to originate membranoproliferative glomerulonephritis-like glomerular lesions. (13)

Paraneoplastic rapidly progressive glomerulonephritis is present in 7 to $9 \%$ of cancer patients, with a prevalence of up to $20 \%$ in patients over 40 years of age. The most related tumors are prostate, bladder, lung, gastric adenocarcinoma, renal cell carcinoma, myelodysplastic and myeloproliferative syndrome and leukemia. It is a serious disease that presents an acute and severe evolution that deteriorates renal function in days or weeks. It is histologically manifested by extracapillary proliferation with crescent formation, and crescent production is stimulated by fibrin accumulation in the Bowman space. (1, 3) Several studies relating its pathophysiology to malignancy have been performed, including unregulated $\mathrm{T}$ cell responses, cytokine production and uteroglobin suppression. Uteroglobin is a protein that under normal conditions is expressed in most epithelia, and it has been shown that in many carcinomas its presence is reduced, experiments with rats have shown that uteroglobin suppression promotes glomerular lesions with fibrin and IgA deposition in 
these rats. The treatment of rapidly progressive paraneoplastic glomerulonephritis is the immediate use of immunosuppressants. $(\mathbf{1}, \mathbf{1 4})$

Paraneoplastic IgA nephropathy has a cancer prevalence of $3 \%$, the tumors most commonly associated with it being those of the respiratory tract, oral cavity, and nasopharynx, where this relationship can be reinforced by the presence of alcoholism, the association has also been described with renal cell carcinoma and lymphoma. It is characterized by microscopic/macroscopic hematuria and / or proteinuria and mesangial proliferation and diffuse IgA deposition in the glomerular mesangium. Light microscopy also helps to reveal an increase in extracellular matrix and hypercellularity in mesangium. $(\mathbf{1}, \mathbf{3}, \mathbf{5}, \mathbf{1 5})$ Its pathophysiology is not well known, but we know that there is a strong link between IgA nephropathy and HLA-DR4, and studies indicate that invasion of the intestinal mucosa by malignancy promotes increased levels. IgA nephropathy may be limited to the kidneys or may be associated with a type of vasculitis, Henoch-Schonlein Purpura (HSP), which is defined by the presence of necrotic skin lesions in the skin. In the absence of cryoglobulins, patients with PHS have an increased risk of malignancy of $5.2 \%$. (1, 3, 16)

And finally, the focal segmental glomerulosclerosis (FSGS) paraneoplastic, which is rarer with few case reports in the literature about its association with cancer . It is associated with lymphoma (particularly Hodking's disease), leukemia, thymoma, hematologic malignancies and non-small cell lung carcinoma. Cancer cells have the ability to synthesize growth factors, and the factor's growth of fibroblasts and transformer beta have been associated with the emergence of FSGS in experimental animal models. $(\mathbf{1 7}, \mathbf{1 8})$ FSGS is defined as involvement of only a few glomerulus (focal) and only one segment of the glomerulus is injured (segmental), with areas of glomerular sclerosis, tubular atrophy and interstitial fibrosis. extracellular matrix containing different types of collagen and laminin, and IgM and C3 deposits are present in a few cases. Treatment can be done with corticosteroids, but this therapy is effective in less than $50 \%$ of patients, so the use of immunosuppressants is also indicated. Immunosuppression should be given as soon as possible, as this condition may progress to renal failure if inadequate treatment is obtained.

\section{(19)}

The choice of paraneoplastic glomerulopathy therapy depends on the etiology, which can be used: low salt diet, water restriction, diuretics, ACE inhibitor, corticosteroids, immunosuppressants (azathioprine, cyclosporine), chemotherapy (in cases of unresectable tumors or metastasis). Complete tumor resolution promotes total resolution of paraneoplastic nephrotic syndrome, but its treatment is complicated in cases of difficult tumor therapy or in cases of metastatic tumors, and the use of corticosteroids and immunosuppressants for nephrotic syndrome is highly debatable, especially in cancer patients. In cases where the association of cancer with glomerulopathy is not very well established. $(\mathbf{1}, \mathbf{2}, \mathbf{1 0}, \mathbf{2 0})$

This study aims to report a case report of a patient with FSGS and metastatic melanoma and the association between segmental and focal glomerulosclerosis (FSGS) and cancer; to analyze possible diagnostic methods available to obtain the association between FSGS in cancer patients and to evaluate the role of immunosuppression as the most appropriate treatment for cancer patients with nephrotic syndrome (NS) .

\section{Clinical case}

Patient 56 years old, married, from Bahia (Brazil), catholic and farmer. Patient sought hospital service in January 2013 due to an ulcerated lesion on the first right toe that had appeared one year ago. A biopsy of the lesion identified invasive malignant melanoma (Breslow $5.8 \mathrm{~mm}$ and Clark V) in the presence of involved margins. After nine days amputation of the right hallux with margin resection was performed. On physical examination he was conscious, feverish. At this time no abnormalities were detected on pulmonary, cardiovascular, abdomen and neurological examination.

After seven months (July 2013) admitted in due to a frame diarrhea, anasarca, dyspnea on little exertion, orthopnea, dry cough, and hematuria. On physical examination, lower limb edema and ascites were identified. Diagnostic hypothesis of nephrotic syndrome made and examinations for investigation of the condition.

The examinations for the diagnosis and research of the above are: urea $57 \mathrm{mg} / \mathrm{dL}$, creatinine $1.22 \mathrm{mg} / \mathrm{dL}$, 
GFR measured at $24 \mathrm{~h}$ urine: $40.0 \mathrm{~mL} / \mathrm{min} / 1.73 \mathrm{~m}^{2}$; uric acid: $6 \mathrm{mg} / \mathrm{dL}, \mathrm{Na}: 142 \mathrm{mEq} / \mathrm{L}, \mathrm{K}: 5.6 \mathrm{mEq} / \mathrm{L}$, bicarbonate: $28 \mathrm{mmol} / \mathrm{L}$, ionic Ca: $4.7 \mathrm{mg} / \mathrm{dL}$; P: $4.2 \mathrm{mg} / \mathrm{dL}$; PTH: $135 \mathrm{pg} / \mathrm{mL}$, Vitamin D: $8 \mathrm{ng} / \mathrm{ml}$, glycemia $79 \mathrm{mg} / \mathrm{dL}$, glycated hb: $5 \%$. And evaluating possible nephrotic syndrome has been identified in proteinuria $10.76 \mathrm{~g} / 24$ hours, albumin serum of $2 \mathrm{~g} / \mathrm{L}$.

For the differential diagnosis of the condition were also dosed : native anti-DNA antibody $16 \mathrm{U} / \mathrm{ml}$, FAN HEP2: homogeneous nuclear standard with titer 1/160 and nucleus 1/160, fibrinogen: $1067 \mathrm{mg} / \mathrm{dL}$, rheumatoid factor: 11, $9 \mathrm{IU} / \mathrm{ml}$, serology for treponema pallidum, hepatitis B and C, HIV: nonreactive, anti-VCA IgM and IgG: nonreactive, complement fraction C3: $147 \mathrm{mg} / \mathrm{dL}$, complement fraction $\mathrm{C} 4: 31.1 \mathrm{mg} / \mathrm{dL}$, immunofixation with absence of anomalous proteins .

Given the need to complete the etiology of NS, a renal biopsy was performed, which revealed the presence of segmental and focal glomerulosclerosis (FSGS) as shown in figures 1, 2 and 3, tip lesion - regenerating acute tubular necrosis (focal) and negative immunofluorescence.

Thus, treatment with prednisone $0.5 \mathrm{mg} / \mathrm{kg}$ and cyclosporine $100 \mathrm{mg} 12 / 12 \mathrm{~h}$ on $08 / 21 / 13$ was instituted during this same period, a reduction in proteinuria $(1 \mathrm{~g} / 24 \mathrm{~h})$ was also identified.

After ten months, she went to the hospital in March 2014 due to the appearance of a foot injury D, which was resected. In October 2014, the patient presented with right inguinal lymph node enlargement. A USG was performed in which: inguinal lymph node with focal cortical thickening, measuring 13x6mm and globular morphological inguinal lymph node, measuring diffuse cortical thickness measuring $15 \mathrm{x} 9 \mathrm{~mm}$. The aspiration lymph node inguinal right it was positive the neoplastic cells. Tests for staging detected on CT chest with micronodule lung pulses, non- specific, neural plate and pleural thickening irregular left and axillary lymph nodes increased in number. Thus, right inguinal lymphadenectomy, right inguinal radiotherapy for four months, right hallux radiotherapy and carboplatin and paclitaxel chemotherapy were instituted.

Due to a new elevation of proteinuria levels with the presence of tumor recurrence, a cyclosporine treatment was prolonged, which was withdrawn only after 19 months of treatment. Cyclosporine withdrawal was performed slowly and was withdrawn on 04/04/2015 when 24-hour proteinuria reached values $<0.4 \mathrm{~g} / \mathrm{L}$ and GFR: $92 \mathrm{ml} / \mathrm{min} / 1.73 \mathrm{~m} 2$.

\section{Discussion}

The case report shows a rare association of FSGS and malignant neoplasia and even more rare is this type of paraneoplastic nephrotic syndrome correlated with the presence of melanoma. In this case, we observed a clear association between the onset of nephrotic syndrome months after the diagnosis of melanoma in the right hallux region, but there was no evidence of metastasis.

Due to the clinical picture of hematuria, anasarca and symptoms resulting from anasarca (orthopnea, dyspnea on exertion), diagnostic tests were performed to confirm the presence of nephrotic syndrome with proteinuria greater than $3.5 \mathrm{~g} / 24$ hours and hypoalbuminemia.

The second step in this case was to identify which the etiology of nephrotic syndrome for better directs treatment. With the extensive complementary investigation, no other factor was identified besides the presence of cancer.

The association of melanoma with this patient's nephrotic syndrome became even more evident when proteinuria worsened, even under cyclosporine treatment, with local tumor recurrence, lymph nodes, and pulmonary metastasis. And the improvement of symptoms when introduced lymphadenectomy and radiotherapy right inguinal and right hallux with chemotherapy adjuvant with carboplatin and paclitaxel.

A therapeutic dilemma in this case was to initiate and maintain cyclosporine in a patient with tumor recurrence to control the paraneoplastic syndrome. Studies indicate that the treatment of FSGS with corticosteroids is effective in less than $50 \%$ of cases and due to this reason, the use of immunosuppressants should associated with corticosteroid. In this patient was used prednisone $0.5 \mathrm{mg} / \mathrm{kg} /$ day and cyclosporine and $100 \mathrm{mg} 12 / 12 \mathrm{~h}$, even though the patient is immunosuppressed due to the neoplastic process. And it has 
been recommended therapy immunosuppressant with established as early as possible with the aim of avoiding or aggravate the development of kidney failure, a result expected in the event of unsuitable treatment of FSGS, which deteriorate the prognosis of the patient.

Cyclosporin withdrawal was performed when the 24-hour proteinuria reached non-nephrotic values confirming the control of FSGS. The patient was discharged with outpatient follow-up, remained asymptomatic. As noted above and exist few studies exposing the outcome d the treatment in cases of paraneoplastic FSGS, and this is due to the rarity of this association. In case it was not possible curative treatment of melanoma, but the control of the disease with the combined use of corticosteroids and cyclosporine, $\mathrm{h}$ hears remission of nephrotic syndrome without the complications associated with these cases and improves the patient's quality of life during the period of melanoma treatment.

\section{Conclusions:}

This case highlights the importance of always investigating in patient's nephrotic syndrome, a paraneoplastic cause, especially when there are associated risk factors. We observed how difficult it is to identify publications that evidence the association between focal segmental glomerulosclerosis (FSGS) and cancer, especially melanoma. And with that, it is still necessary to develop serum biomarkers and histological evidence that the associations between the development of FSGS in cancer patients. Regarding the controversial role of immunosuppression in these cases, especially if the disease is relapsed or if the patient needs chemotherapy, it still needs to be individualized on a case by case. In this patient there was a clear benefit in immunosuppression, especially in preserving renal function and promoting control of nephrotic syndrome adjuvant to tumor treatment.

\section{References:}

1. Bacchetta J, Juillard L, Cochat P, Droz J. Paraneoplastic glomerular diseases and malignancies. Critical Reviews in Oncology / Hematology. 2009; 70: 39-58.

2. Cohen EP, Krzesinski J, Launay-Vacher V, ET AL. Onco-Nephrology: Core Curriculum 2015. American Journal of Kidney Diseases. 2015; 66 (5): 869-883.

3. Cambier J, Snore P. Onco-Nephrology: Glomerular Diseases with Cancer. Clinical

4. Audardi V, Larousserie F, Grimbert P, ET AL. Minimal change nephrotic syndrome and classical Hodgkin's lymphoma: Report of 21 cases and review of the literature. Kidney International. 2006; 69: 2251-2260.

5. Fawole A, Daw H, Tayor H, Rashidi A. Immunoglobulin A Nephropathy Associated with Mesothelioma. Wiscosin Medical Society. 2012; 111: 29-33.

6. Guabello G, Brunetii L, Palladini G, ET AL. Paraneoplastic Cushing's Syndrome and Nephrotic Syndrome in a Patient With Disseminated Small Cell Lung Cancer. American Journal of Clinical Oncology. 2008; 31: 102-103.

7. Glassock RJ. Attending Rounds: An Older Patient with Nephrotic Syndrome. Clinical Journal of the American Society of Nephrology. 2012; 7: 665-670.

8. Takeda S, Chinda J, Murakami T, ET AL. Development of features of glomerulopathy in tumor-bearing rats: a potential model for paraneoplastic glomerulopathy. Nephrology Dialysis Transplantation. 2011; $0: 1-7$.

9. Iwanaga N, Kamachi M, Fujikawa K, ET AL. Membraneous Glomerulonephritis and Non-Hodgkin's Lymphoma in a Patient with PrimarySjogren's Syndrome. Internal Medicine. 2007; 46: 191-194.

10. Faur D, Martin N, Archuleta JM, Torguet P. A Man With Nephrotic Syndrome and a Mediastinal Mass. American Journal of Kidney Diseases. 2012; 60: A25-A27.

11. Monga D, Jhaveri KD. Chapter 6: Glomerular Diseases and Cancer. American Society of Nephrology. 2016: 1-9.

12. Yong TY, Li JYZ, Khow K. Minimal change disease as a paraneoplastic manifestation of solid malignant tumors. Nephrology Reviews. 2012; 4: 27-32.

13. Gupta K, Nothing R, Das A, Kumar MS. Membranoproliferative glomerulonephritis in a carcinoma with unknown primary: An autopsy study. Indian Journal of Pathology and Microbiology. 2008; 51: 
230-233.

14. Vietinghoff SV, Schneider W, Luft FC, Kettritz R. Crescentic glomerulonephritis and malignancy-guilt or guilt by association? Nephrology Dialysis Transplantation. 2006; 21: 3324-3326.

15. Kocyigit I, Dortdudak S, Eroglu E, ET AL. Immunoglobulin A nephropathy could be a clue for the recurrence of gastric adenocarcinoma. Nephrology (Madrid.) 2013; 33 (6): 849-67.

16. Bergmann J, Buchheidt D, Waldherr R, ET AL. IgA Nephropathy and Hodgkin's Diseases: A Rare Coincident. American Journal of Kidney Diseases. 2005; 45: E16-E19.

17. Yamauchi J, Ubara Y, Suwabe T. Segmental Focal Glomerulosclerosis Associated With Invasive Thymoma. TherapeuticApheresisandDialysis. 2011; 5: 210-211.

18. Loi S, Perry GJ, Standish H, Dowling J. Glomerulosclerosis: A paraneoplastic phenomenon ? Nephrology. 2004; 9: 387-391.

19. Aggarwal N, Batwara R, McCarthy ET, ET AL. Serum Permeability Activity in Steroid-Resistant Minimal ChangeNephrotic Syndrome Is Abolished by Treatment of Hodgkin Disease. American Journal of Kidney Diseases. 2007; 50: 826-829.

20. Tucci M., Stucci S., Vallarelli S., ET AL. Paraneoplastic Segmental Focal Glomerulosclerosis in Sarcomatoid Renal Cell Cancer. Journal of Clinical Oncology. 2015; 33: 66-70.

\section{Figures:}

Figure 1: Glomerulus with segmental and focal sclerosis in patient with melanoma, stained by Schiff's periodic acid method. (400x magnification)

Figure 2: Glomerulus with segmental and focal sclerosis in patient with melanoma, stained by the Schiff's periodic acid method. (400x magnification)

Figure 3: Glomerulus with segmental and focal sclerosis in patient with melanoma, stained by the periodic acid-silver methenamine (PAMS) method. 400x magnification 


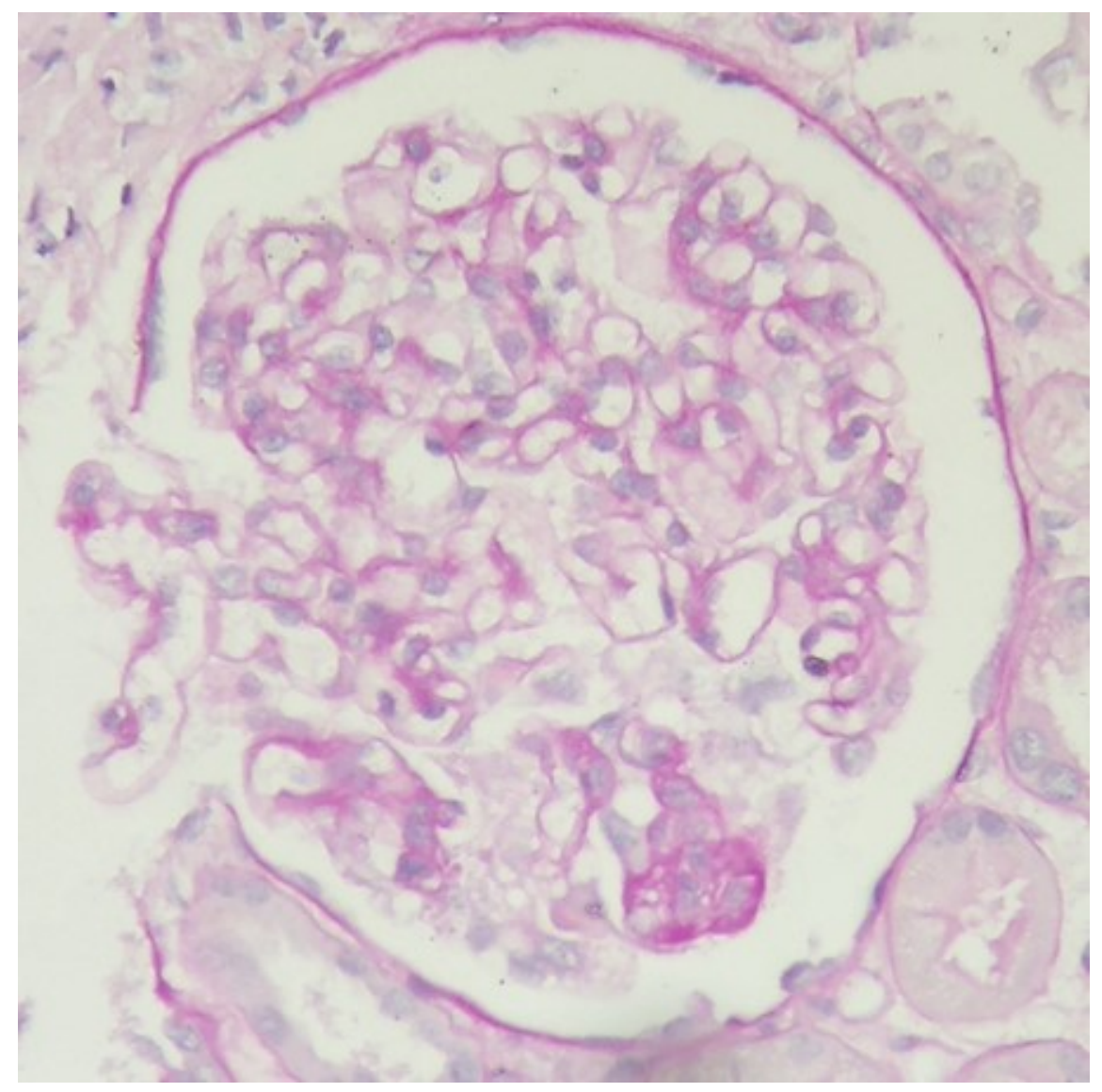




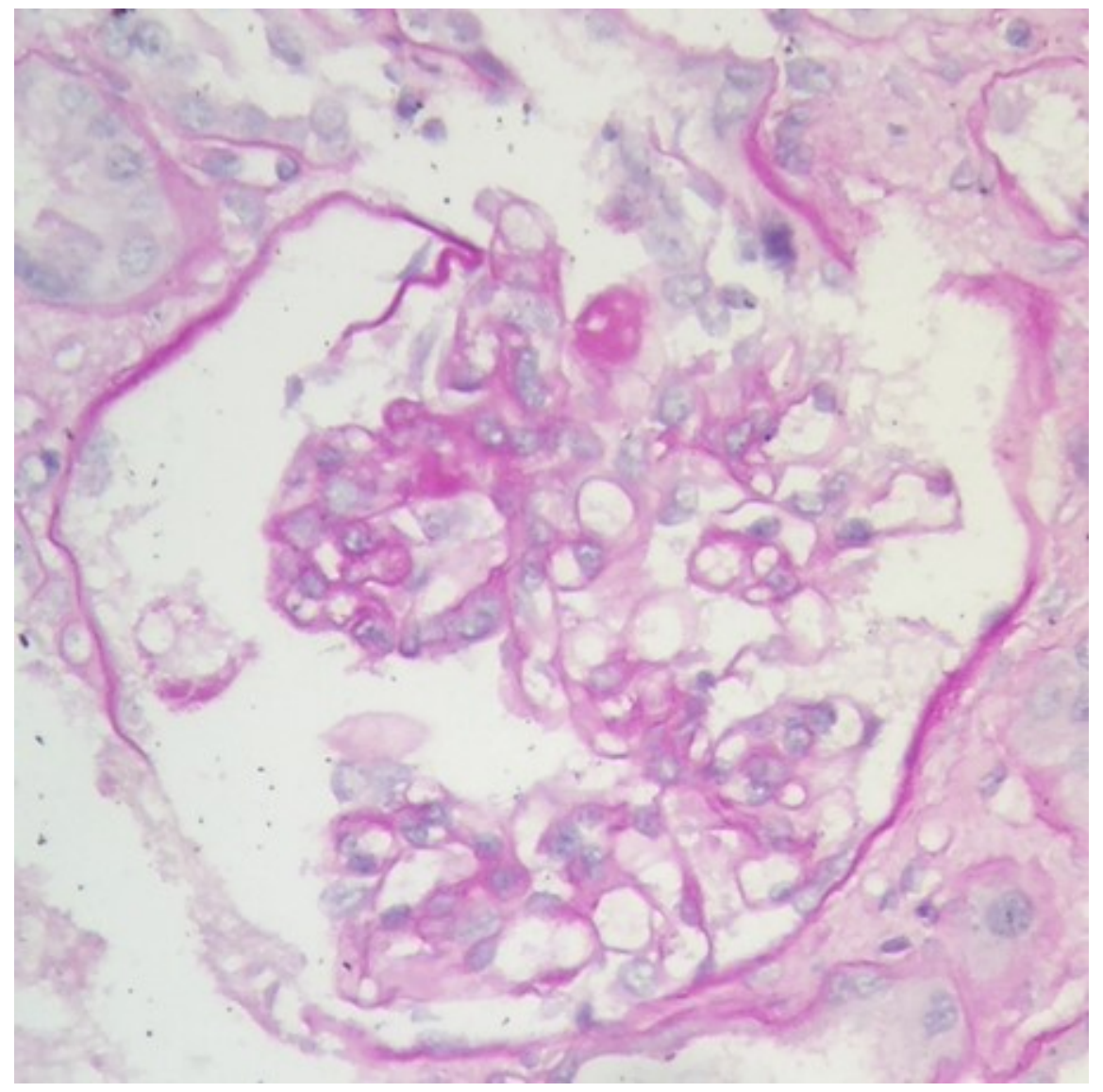




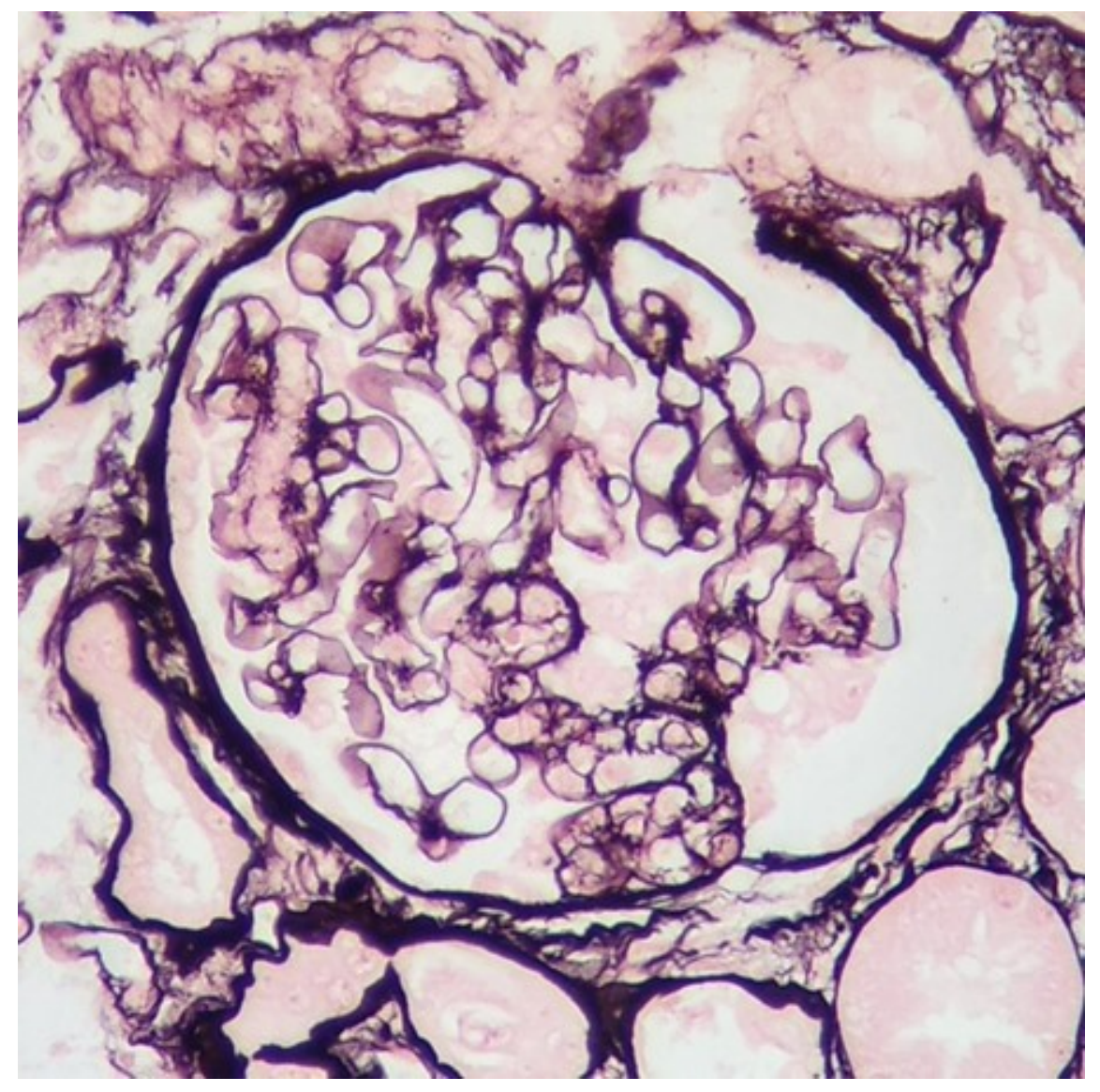

